\title{
Normal and anomalous diffusion of non-interacting particles in linear nanopores
}

\author{
S. Zschiegner ${ }^{1,2}$, S. Russ ${ }^{2,3}$, R. Valiullin ${ }^{1}$, M.-O. Coppens ${ }^{4,5}$, A.J. Dammers ${ }^{4}$, \\ A. Bunde ${ }^{2}$, and J. Kärger ${ }^{1, \text { a }}$ \\ 1 Universität Leipzig, Experimentelle Physik I, Linnéstraße 5, 04103 Leipzig, Germany \\ 2 Universität Giessen, Theoretische Physik III, Heinrich-Buff-Ring 16, 35392 Giessen, Germany \\ 3 Freie Universität Berlin, Physik, Arnimallee 14, 14195 Berlin, Germany \\ 4 TU Delft, DelftChemTech, Julianalaan 136, 2628 BL Delft, The Netherlands \\ ${ }^{5}$ Rensselaer Polytechnic Institute, Chemical and Biological Engineering, 110 8th Street, Troy, \\ NY 12180, USA
}

\begin{abstract}
The diffusion of gas molecules in pores is determined by the collisions between the molecules as well as by the collisions of the molecules with the pore walls. In many applications the so-called Knudsen regime is of particular interest. In this regime the collisions of the molecules with the pore walls play the crucial role, while the inter-molecular collisions can be neglected. Here we study the influence of surface roughness on the coefficients of self (or tracer) diffusion and transport diffusion. Considering the first four iterations of a generalised fractal Koch surface, we construct pore models of different roughness. For these model pores we have performed detailed simulations of both diffusion coefficients using a cube-based algorithm. The molecular trajectories can be mapped onto Lévy walks to determine the diffusion properties. In linear two-dimensional (2d) channels we observe anomalous diffusion, which can also be induced in smooth and rough three-dimensional (3d) pores by anomalous reflection laws. Normal diffusion is found in convoluted $2 \mathrm{~d}$ pores and in all $3 \mathrm{~d}$ pores when a diffuse reflection law is applied.
\end{abstract}

\section{Introduction}

Diffusion of gases in disordered and porous media has been extensively studied for decades [1-8] with applications in heterogeneous catalysis [9], adsorption [10] and separation [11]. Recent progress in synthesising nanostructured porous materials $[10,12,13]$ has provided essentially unlimited options for the generation of purpose-tailored pore architectures. Therefore, there is an increasing demand for clarification of the main features of molecular transport in such systems $[14,15]$. The performance of chemical conversion by heterogeneous catalysis and of chemical separation by molecular sieving and selective adsorption with nanoporous materials may be notably enhanced by the application of materials with a bimodal pore structure. In addition to micropores or narrow mesopores (nanopores) as the location of chemical separation and conversion, such materials are traversed by a network of large mesopores or macropores. These transport pores ensure fast exchange of the molecular species involved with the surrounding fluid, and thus reduce the impairing influence of the finite rate of molecular transport on the overall performance [13]. Mesopores give rise to a number of most attractive phenomena which are typical of mesoscopic systems. They in particular include the occurrence of adsorption hysteresis, i.e., the fact that for a given temperature and external pressure, depending on

\footnotetext{
a e-mail: kaerger@physik.uni-leipzig.de
} 


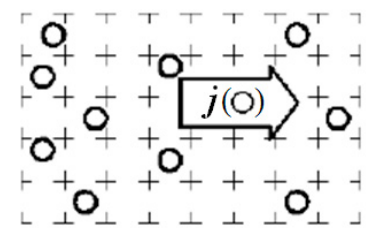

(a)

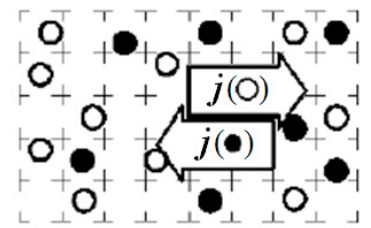

(b)

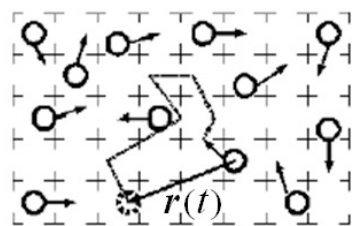

(c)

Fig. 1. Microscopic situation corresponding to the measurement of transport diffusion (a) and self diffusion (b, c). The flux of the 'labelled' molecules (open symbols) in (b) is counterbalanced by that of the 'unlabelled' molecules (filled symbols). If the mobility of the unlabelled molecules is unaffected by the presence of the labelled molecules, the fluxes in (a) and (b) are equal and the transport and self-diffusivities coincide $[3,21]$.

the history, the total amount of guest molecules may have quite different values that appear to remain invariable over very large time spans [16]. Most interestingly, this situation has been found to occur irrespective of the fact that the rate of molecular diffusion was large enough to ensure sorbate equilibration over much shorter intervals of time [12]. In Ref. [17] this extremely slow process of relaxation towards the absolute minimum in the free energy through a series of local minima has been attributed to the necessity of equilibration of particle ensembles rather than of individual molecules. Similarly, mesopores have been found to lead to pronounced shifts in the critical temperature to lower values [18] as well as to well-defined decreases in the freezing temperature [19]. For low external pressures, molecular concentrations within the mesopores become sufficiently small so that molecular propagation is dominated by Knudsen diffusion, where the interaction of the molecules with the pore walls plays the crucial role and the intermolecular interactions can be neglected. It is this situation, to which this contribution is dedicated.

If intermolecular collisions may be neglected, the molecules perform a sequence of free flights, and change direction statistically after each collision with the pore wall. Depending on the experimental conditions, two kinds of diffusion can be distinguished. So-called transport diffusion arises in a non-equilibrium situation, in which the particles diffuse from one side of the system to the opposite side, under the influence of a gradient in concentration (and hence chemical potential). On the other hand, self (or tracer) diffusion occurs under equilibrium conditions. These processes are described by the transport diffusion coefficient $D_{t}$ and the self (or tracer) diffusion coefficient $D_{s}$. The transport diffusivity $D_{t}$ is defined by Fick's First Law as the proportionality constant between the current density $j$ and the concentration gradient $d c / d x$ :

$$
j=-D_{t} d c / d x
$$

while $D_{s}$ is usually defined, via the Einstein equation, by the mean-square displacement $\left\langle x^{2}(t)\right\rangle$ of a random walker

$$
\left\langle x^{2}(t)\right\rangle=2 d D_{s} t
$$

after a long time $t$, where $d$ is the embedding dimension of the pore $(d=2$ or 3$)$.

If the self-diffusivity as defined by Eq. (2) turns out to be independent of time by application of the central limit theorem [3,20] the definition via Eq. (2) may be shown to be completely equivalent to a definition on the basis of Eq. (1), where now $j$ and $c$ refer to the flux and concentration of labelled molecules (e.g., the open spheres in Fig. 1(b)) and the factor of proportionality is now the self (or tracer) diffusivity $D_{s}$ rather than $D_{t}[3,21]$. Under these conditions, the probability of molecular displacements in the $x$-direction approaches a Gaussian, with the molecular mean-square displacement characterising the distribution width linearly increasing with the observation time. This, however, is exactly the message of the Einstein relation.

Let us now compare the microdynamic situations in experiments, in which the transport diffusion (Fig. 1(a)) and the self diffusion (Figs. 1(b) and (c)) are investigated. Let us in particular compare the fluxes (of the molecules represented by empty circles) in Figs. 1(a) and (b). Generally, the molecules diffusing in Fig. 1(a) from left to right, i.e., propagating from regions of higher to lower concentration, will experience a situation different from the one 
shown in Fig. 1(b), where the concentrations remain uniform. Not unexpectedly, therefore, the molecular fluxes (and hence also the corresponding diffusivities, since we have implied identical concentration gradients) under the conditions of transport diffusion and self diffusion, may be different. This is the situation well known, e.g., from the study of zeolitic adsorbate-adsorbent systems [2-4]. By the same chain of arguments one has to conclude that the coefficients of transport diffusion and of self diffusion have to coincide if the interaction between the particles is negligibly small, as in the case of Knudsen diffusion. Because of this the microdynamic situations in Figs. 1(a) and (b) are identical. This includes both the coefficients and the asymptotic behaviour of Eqs. (1) and (2). The present paper is dedicated to the exploration of this situation by dynamic simulations and analytic considerations.

After laying down the details of our implemented diffusion algorithm in the following section, we show results from simulations of $2 \mathrm{~d}$ and $3 \mathrm{~d}$ channels of different roughness in section 3 . The results indicate that the coefficients of transport diffusion and self diffusion coincide perfectly. We also hint on possible pitfalls when implementing these simulations, since deviations of the applied reflection law may lead to anomalous diffusion behaviour. We conclude in section 4 with an encouragement to the experimentalists to take profit of the most recently provided options to fabricate nanotubular materials $[12,19,22,23]$ and to confirm the predicted results experimentally.

\section{Diffusion algorithm}

Usually, a direct approach is used to calculate collisions of particles with pore walls. Using such a method, every part of the wall has to be checked for possible hits by the particle. For systems with simple geometries and only a few different wall segments, especially in $2 \mathrm{~d}$, such a direct approach may be sufficient (indeed, we used this approach in the 2d calculations in [24]). However, for systems with many wall segments, even an optimised wall sorting scheme that searches for possible collisions in order of ascending distance of the wall from the starting point of the particle (often used in 3d graphics) becomes too time-consuming. In our algorithm, we use cubic elements ("cubicles") to generate the pore structure. Particle trajectories are calculated as successive short-path elements through adjoining cubicles. Since only those cubicles are needed that are actually crossed by the particle, the calculation time scales in a strictly linear way with the system size. Hence, large systems can easily be implemented using our simulation method.

\subsection{Pore structure}

Our pore system (see Fig. 2) is based on a periodic cubic lattice of size $c_{x, \max } \times c_{y, \max } \times c_{z, \max }$, consisting of elementary cubicles. The cubicles forming the porous structure can be either empty, i.e., part of the inner pore, or solid, i.e., part of the pore walls. Clearly, the particles only move inside the empty cubicles. If a particle hits a cubicle belonging to the wall, it is reflected back into the empty part of the inner pore, where it can move freely. The location of any given particle is represented by three different sets of coordinates, i.e., by the "house number" $n$ that summarises the cubicles inside a certain region (compare Fig. 2), the cubicle vector $\boldsymbol{c}=\left(c_{x}, c_{y}, c_{z}\right)$ to the centre of the given cubicle in the given "house" and the incubicle coordinate $\boldsymbol{r}=\left(r_{x}, r_{y}, r_{z}\right)$ inside the cubicle, with $-1 \leq r_{x}, r_{y}, r_{z} \leq 1$, and the geometrical centre at $r_{x}=r_{y}=r_{z}=0$. Trajectories within individual cubicles are calculated using basic mathematical formulas, and the trajectories through neighbouring cubicles are combined to form the complete flight path. The particle velocity is given by $\boldsymbol{v}=\left(v_{x}, v_{y}, v_{z}\right)$, where the absolute value $v$ is kept constant during our simulations.

Diffusion of particles through the above described pores is completely based on the underlying hierarchy of house numbers and cubicles. Only trajectories inside the cubicles are calculated. Whenever a particle leaves such a cubicle, it enters a neighbouring one, and the next part of the trajectory is determined. Since this algorithm is lattice-based instead of time-based, it is mandatory to keep track of the time. An example to extract discrete time steps is discussed below. 


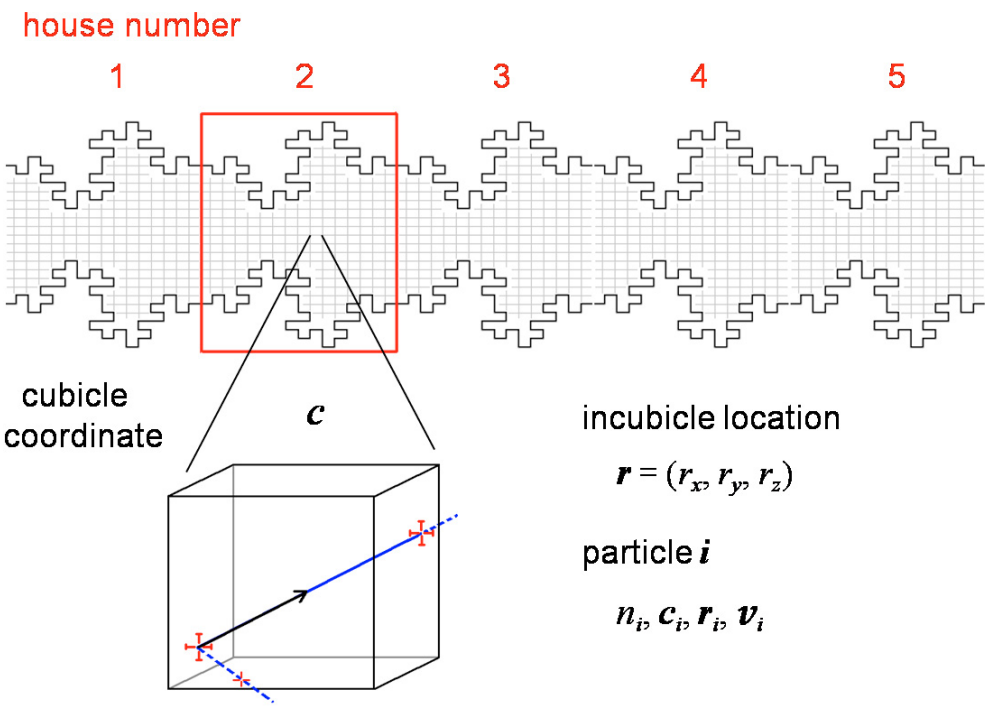

Fig. 2. The framework of the simulation. The pore system is based on a coordinate hierarchy: Empty or filled cubicles determine the local geometry of the pore. They are embedded into a superstructure of house numbers which repeat the local geometry throughout the pore length. The location of a particle $i$ is described by its house number $n_{i}$, cubicle coordinate $\boldsymbol{c}_{i}$ and incubicle coordinates $\boldsymbol{r}_{i}$.

(a)

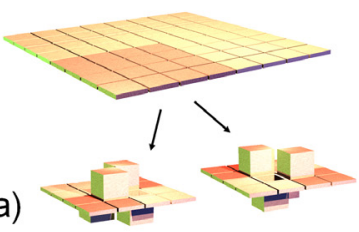

(b)

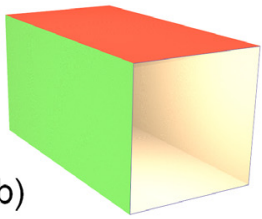

(c)

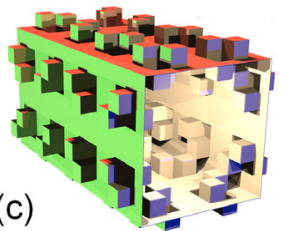

(d)

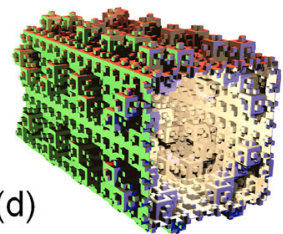

Fig. 3. (Colour online) examples of pore geometries. (a) Volume conserving and surface doubling random iterator used to generate rough surfaces from (b) the basic smooth pore; (c) and (d) show the $1^{\text {st }}$ and $2^{\text {nd }}$ iteration, respectively.

Every cubicle of the pore system can be generated individually, giving rise to a whole variety of shapes, ranging from simple linear pores with a smooth surface to percolation clusters. In our studies [24-28] we used different iterations of a generalised Koch surface. For this task we applied a random iterator that is shown in Fig. 3(a). It is designed to be volume conserving, while doubling the surface with every generation. Figures $3(\mathrm{~b})-(\mathrm{d})$ show pores with different roughnesses that were generated using this iterator.

\subsection{Dynamics}

From the exact location and velocity of a particle after $j$ steps $\left(n_{j}, \boldsymbol{c}_{j}, \boldsymbol{r}_{j}, \boldsymbol{v}_{j}\right)$, given by the house number, cubicle coordinate, and location within the cubicle, it is possible to determine the next location where the particle will hit the surface of this cubicle, using the intercept theorem from basic mathematics. On that basis, it is determined if the particle is reflected, enters the adjacent cubicle, or even enters the next house number. Depending on entry point and flight direction three walls of the cubicle are possible candidates for the next collision. To determine the actual wall hit by the particle, one has to calculate the time to get from the current location $\boldsymbol{r}=\left(r_{x}, r_{y}, r_{z}\right)$ to all three (infinite) bounding planes, embedding the three possible wall elements. The minimal time $t^{*}$ to reach one of those bounding planes is [29]:

$$
t^{*}=\min \left[t_{x}=\left(v_{x} /\left|v_{x}\right|-r_{x}\right) / v_{x}, t_{y}=\left(v_{y} /\left|v_{y}\right|-r_{y}\right) / v_{y}, t_{z}=\left(v_{z} /\left|v_{z}\right|-r_{z}\right) / v_{z}\right] .
$$


The two other bounding planes would be crossed outside of the current cubicle, so the time to reach them can be discarded. The new incubicle coordinates $\boldsymbol{r}^{\prime}=\left(r_{x}^{\prime}, r_{y}^{\prime}, r_{z}^{\prime}\right)$ of the particle will then be:

$$
r_{x, y, z}^{\prime}=r_{x, y, z}+v_{x, y, z} t \quad \text { or } \quad \boldsymbol{r}^{\prime}=\boldsymbol{r}+\boldsymbol{v} t .
$$

Accordingly, the simulation time has to be increased by $t^{*}$. The component of $\boldsymbol{r}^{\prime}$ which is on the boundary of the present cubicle always has an integer value of \pm 1 . It can directly be used to determine the adjacent cubicle $\boldsymbol{c}^{\prime}$ on whose boundary the particle is located now:

$$
c_{x, y, z}^{\prime}=c_{x, y, z}+\operatorname{int}\left(r_{x, y, z}^{\prime}\right) .
$$

This simple formula includes every possibility of entry point and flight direction by exploiting the properties of the range of the incubicle coordinate $\boldsymbol{r}^{\prime}$. Including periodic boundary conditions in the calculation of $\boldsymbol{c}^{\prime}$, this equation is changed to

$$
c_{x, y, z}^{\prime}=\left(c_{x, y, z}+\operatorname{int}\left(r_{x, y, z}^{\prime}\right)+c_{x, y, z, \max }\right) \bmod c_{x, y, z, \max } .
$$

Hereby the house number has to be increased or decreased appropriately. Now, two cases have to be taken into account. If $\boldsymbol{c}^{\prime}$ is empty, the particle starts the next computation step in the new cubicle $c_{x, y, z}=c_{x, y, z}^{\prime}$ with the new incubicle coordinates $r_{x, y, z}=r_{x, y, z}^{\prime}-2 \operatorname{int}\left(r_{x, y, z}^{\prime}\right)$. This way, the coordinate that shares the boundary between old and new cubicle inverts its sign, while the other coordinates remain unchanged. If $\boldsymbol{c}^{\prime}$ is filled, the starting point of the next computation step is the end point of the previous one, $r_{x, y, z}=r_{x, y, z}^{\prime}$. In this case, a new velocity vector through the cubicle has to be chosen according to an appropriate reflection law.

The particles are emitted thermally from the surface. Therefore, they leave with an angle $\vartheta \in[-\pi / 2, \pi / 2]$ to the normal component of the surface that is governed by Lambert's cosine law. Hereby the reflection angle $\vartheta$ occurs with a probability $P(\vartheta, \varphi) \propto \cos \vartheta d \Omega$. The solid angle $d \Omega$ is equal to $d \vartheta$ in $2 \mathrm{~d}$, and equal to $\sin \vartheta d \vartheta d \varphi$ in $3 \mathrm{~d}$. This leads to the same luminance in every direction. If the solid angle $d \Omega$ is not taken into account correctly, anomalous diffusion effects may arise. It is shown later how this can be used to test the correct implementation of Lambert's law.

\subsection{Self (or tracer) diffusion}

To calculate the self (or tracer) diffusivity we need to keep track of time, since for every discrete time $t_{i}$ the mean-square displacement $\left\langle x^{2}\left(t_{i}\right)\right\rangle$ has to be calculated. This is accomplished by linear interpolation within the cubicles. Every calculation step starts at a certain time $t$ at $r_{x, y, z}$ and ends at a time $t^{\prime}=t+t^{*}$ at $r_{x, y, z}^{\prime}$. If $\operatorname{int}(t)<\operatorname{int}\left(t^{\prime}\right)$ the simulation has advanced more than one time step since the last measurement. In this case the exact location at all intermediate discrete times has to be determined. Depending on the flight path, within the cubicle more than one discrete time step may have passed, which has to be taken into account accordingly. From these discrete measurements of $\boldsymbol{r}$, the squared displacement $x^{2}\left(t_{i}\right)$ can easily be averaged, leading to the self-diffusion coefficient $D_{s}$. Note that this method to extract discrete time steps can also be used for other measurements, e.g., relaxation into stationarity or the following corrected method to determine the transport diffusion coefficient $D_{t}$.

\subsection{Transport diffusion}

In the simulations of the transport diffusion coefficient $D_{t}$, a concentration profile, $c(x)$, along the pore length $L$ is applied with imposed concentrations $c=c_{0}=1$ on the left hand side and $c=0$ on the right hand side $(x=L)$. All particles start at $x=0$, which means that $n_{0}=0, c_{x, 0}=0, r_{x, 0}=-1$, with a velocity $v_{0}$, uniformly distributed into all directions. Then, they perform a random walk in $2 \mathrm{~d}$ or $3 \mathrm{~d}$, according to the chosen reflection law. They are absorbed when they hit either the left boundary $(x=0$, where they entered) or the right boundary at $x=L\left(n_{i}=n_{\max -1}, c_{x, i}=c_{x, \max }, r_{x, i}=+1\right)$, which corresponds to Dirichlet boundary conditions. After some relaxation time, this leads to a constant current density $j$, as 
(a) step

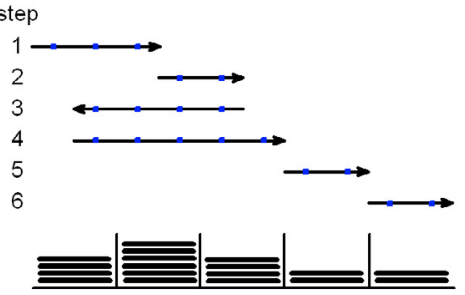

(b)

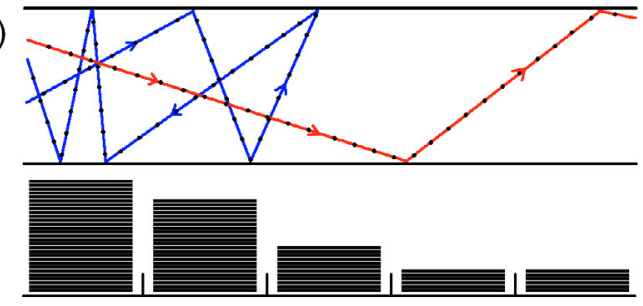

Fig. 4. Schematics for generating the particle histogram and, hence, $c(x)$ for one particle trajectory in $1 \mathrm{~d}$ (a) and for two particle trajectories in $2 \mathrm{~d}(\mathrm{~b})$. The histograms in the lower part are incremented at the actual location after each time step.

described above. Since relaxation of a particle flow into a stationary state is usually very timeconsuming, as the particle flux has to be monitored throughout the system, we use a method proposed by Evans et al. [30] that we extended in [28]. The particle flux $j_{x}$ in the $x$-direction is derived from the (transmission) probability $f_{t}$ that a particle starting at the left boundary will leave the pore through the right boundary:

$$
j_{x}=c_{0} f_{t}\left\langle v_{x}\right\rangle
$$

where $\left\langle v_{x}\right\rangle$ is the mean velocity in $x$-direction. Accordingly, for calculating $f_{t}, N$ random trajectories are considered that start at $x=0$ and end when either $x=0$ or $x=L$ is reached. Combining this with Fick's law (Eq. (1)) yields:

$$
D_{t}=-c_{0} f_{t}\left\langle v_{x}\right\rangle(d c / d x)^{-1} \text {. }
$$

Usually, the concentration gradient is assumed to be constant and equal to $d c / d x=-c_{0} / L$. Then these equations combine to $D_{t}=f_{t}\left\langle v_{x}\right\rangle L$.

A problem arises if the concentration gradient $d c(x) / d x$ within the pore is not well approximated by a constant. This can be due to simple entrance effects, as shown in [28], where the $f_{t}$ method was extended to correct for this. To obtain the correct value of $D_{t}$, we need the concentration $c(x)$ within the pore and the associated concentration gradient $d c(x) / d x$. For this purpose we need to determine the exact location for each particle after every integer time step. The discrete times can be easily extracted through linear interpolation as shown in the previous section. Figure 4 shows the schematics of such a procedure in $2 \mathrm{~d}$, where $c(x)$ is calculated from the trajectories used to calculate $f_{t}$. The particle positions at every discrete time step are indicated by dots. Including all particles at all time steps, after normalising by the particle number this becomes the time-averaged concentration $c(x)$, which is the ensemble averaged concentration $c(x)$, if the problem is ergodic (see lower part of Fig. 4) [31]. The corresponding concentration gradient is then substituted into Fick's law (Eq. (1)) or Eq. (8), respectively.

\section{Results}

Reflecting the randomness of molecular propagation, diffusion is a complex phenomenon by its very nature. In the following, we are considering some of the main features of Knudsen diffusion in channel pores. First we consider the probability distribution of jump lengths inside the pores. Using this, we try to predict the diffusion behaviour from comparisons with 1d Lévy walks $[5,32-34,36]$. This is followed by the examination of prerequisites of normal and anomalous diffusion in 3d and 2d. Finally we check our considerations using one distinct pore geometry that can be approached both in simulations and analytically [35].

\subsection{Distribution of jump lengths}

The first quantity we are interested in the distribution $P(|x|)$ of the jump lengths $|x|$ between successive collisions parallel to the channel. Figure 5 shows that asymptotically $P(|x|)$ decays 


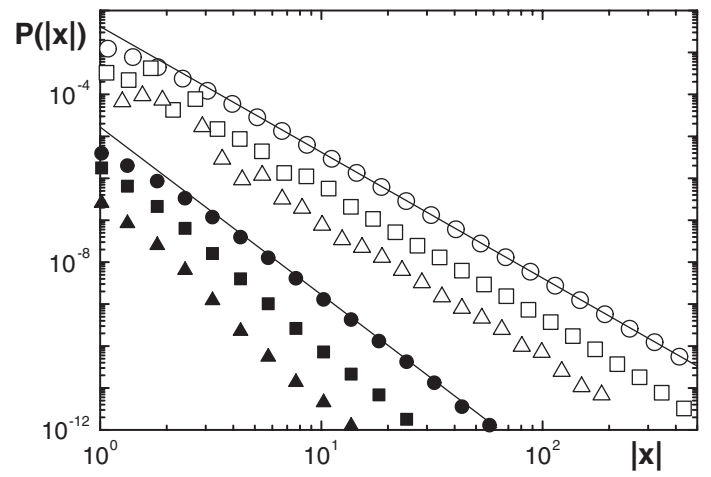

Fig. 5. The distribution $P(|x|)$ is plotted versus $|x|$ for $2 \mathrm{~d}$ pores (open symbols) and for $3 \mathrm{~d}$ pores (filled symbols) with smooth surface (circles), $1^{\text {st }}$ iteration (squares), and $3^{\text {rd }}$ iteration (triangles) rough surface. The $3 \mathrm{~d}$ data have been shifted down by a factor of 1000 . The lines of slope -3 and -4 are guides to the eye.

as $P(|x|) \propto|x|^{-(1+\beta)}$, with $\beta=2$ in $2 \mathrm{~d}$ and $\beta=3$ in $3 \mathrm{~d}$, irrespective of the roughness under the condition of diffuse reflection. This asymptotic Lévy walk behaviour determines the diffusion properties.

In our simulations the velocity is constant, so the time of each jump is proportional to the jump length $l$. For very large jumps, we have $x \approx l$, and the jump time $t$ is proportional to the jump length in $x$-direction. In $2 \mathrm{~d}$, large jumps occur close to the angles $\vartheta= \pm \pi / 2$ (measured to the normal component of the surface) and are thus quite frequent. In $3 \mathrm{~d}$, the jump length is determined by two angles, $\vartheta$ and $\varphi$, where only the proper combination of both leads to very large jumps. Naturally, this combined probability of both angles is quite small, leading to very rare large jumps and $\beta=3$.

It is well known $[32-34,36]$ that for a Lévy walk, the self-diffusion coefficient $D_{s}(t)$ as introduced by Eq. (2) scales as

$$
D_{s}(t)=D_{s}^{0} \ln t \quad \text { for } \quad \beta=2
$$

and

$$
D_{s}(t)=D_{s} \quad \text { for } \quad \beta=3
$$

with the constants $D_{s}$ and $D_{s}^{0}$. Accordingly, we expect that in $2 \mathrm{~d}$, where $\beta=2$, the diffusion is anomalous with a diffusion coefficient that tends to infinity with increasing time $t$. Due to the time-dependence of $D_{s}$, it is essential to keep $t$ fixed when comparing coefficients of different $2 \mathrm{~d}$ pores. It has been shown in [27] by scaling arguments that the logarithmic correction $\ln t$ of Eq. (9) transforms into a corresponding logarithmic correction of $\ln L$ for the $2 \mathrm{~d}$ transport diffusion coefficient $D_{t}$. In $3 \mathrm{~d}, \beta=3$ and we expect normal diffusion since large jumps are rare (see above). We would like to note that for smooth $2 \mathrm{~d}$ pores, the logarithmic time-dependence of $D_{s}$ can be directly obtained from Lambert's cosine law [24,27,37].

From the jump length distribution alone, a decrease in diffusivity with increasing roughness can be predicted. Obviously rougher pores show an increased probability of small jumps that originate in the smaller details of the pore structure where single trajectories may be reflected. As an effect, in rough pores long flights become less frequent.

\subsection{Normal diffusion in $3 d$}

In $3 \mathrm{~d}$ pores two angles determine the particle trajectories. As a result, the influence of jumps parallel to the pore axis as generated by Lambert's law becomes sufficiently small, giving rise to normal diffusion. Figures 6(a) and (b) show the results of our self diffusion and transport diffusion simulations in smooth and rough Koch-shaped pores, scaled to directly show the associated diffusion coefficients $D_{s}$ and $D_{t}$. The diffusivity decreases with increasing surface 

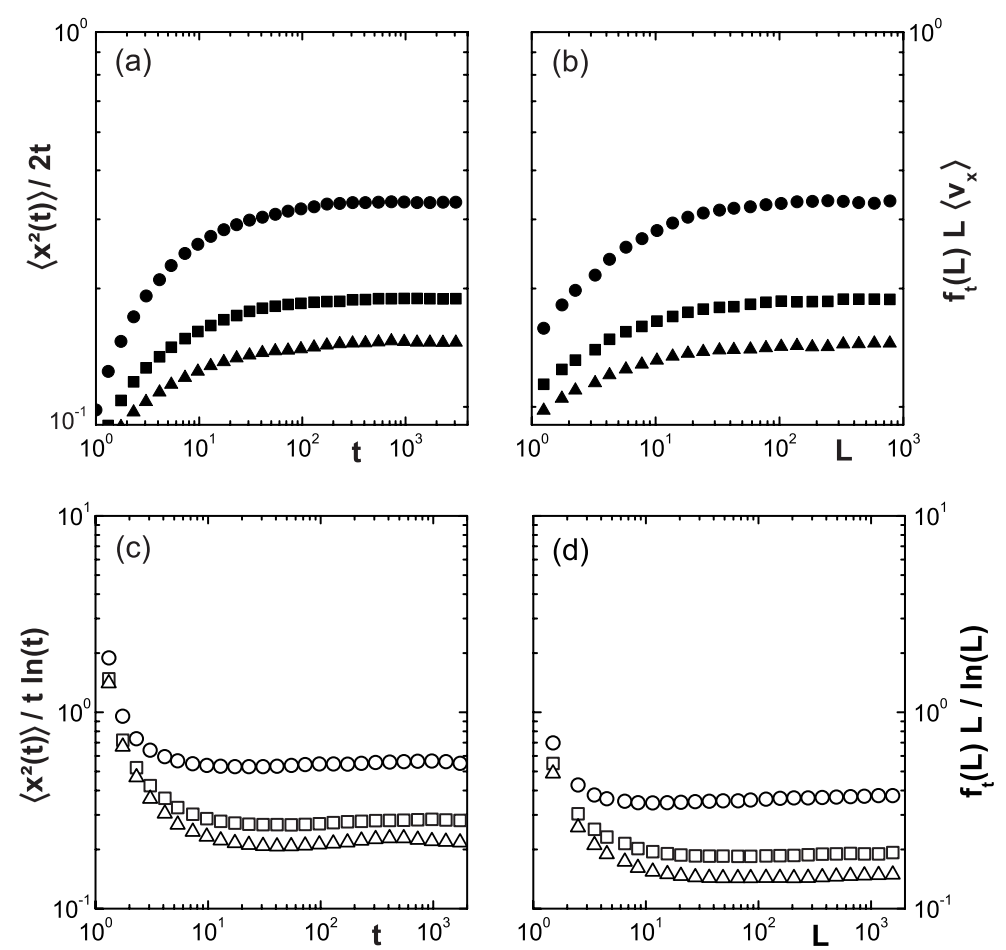

Fig. 6. The (scaled) mean-square displacements $\left\langle x^{2}(t)\right\rangle$ and transmission probability $f_{t}$ resulting from numerical simulations in $3 \mathrm{~d}$ and $2 \mathrm{~d}$ pores with different surface roughness, namely in smooth pores (circles) and after the $1^{\text {st }}$ (squares) and $3^{\text {rd }}$ (triangles) iteration. (a) Self-diffusivity $D_{s}=\left\langle x^{2}(t)\right\rangle /(2 t)$ and (b) transport diffusivity $D_{t}=f_{t} L\left\langle v_{x}\right\rangle$ for $3 \mathrm{~d}$ pores both become asymptotically constant. In $2 \mathrm{~d}$, the mean-square displacement (c) scales as $\left\langle x^{2}(t)\right\rangle \propto t \ln t$, while the transmission probability $f_{t}(\mathrm{~d})$ scales as $f_{t} \propto \ln L / L$.

roughness $[7,28]$. Since the data points reach a plateau for large times $t$, normal diffusion is confirmed for both diffusion mechanisms in smooth and in rough pores.

Most remarkably, the occurrence of normal diffusion in $3 \mathrm{~d}$ channel pores is the result of the particle reflections obeying Lambert's law $(P(\vartheta, \varphi) \propto \cos \vartheta d \Omega$; Fig. 7, centre). A homogeneous angular distribution $(P(\vartheta, \varphi) \propto d \Omega$; Fig. 7, left) results in anomalously fast diffusion in smooth pores. This effect weakens with increasing roughness since the trajectories parallel to the pore axis are blocked. Pronounced vertically reflected particles $(P(\vartheta, \varphi) \propto \cos \vartheta d \vartheta d \varphi$; Fig. 7, right) diffuse anomalously fast only in rough pores, where long trajectories start at pore wall elements perpendicular to the pore axis. Since these wall elements are absent in smooth pores, normal diffusion is observed in this case.

\subsection{Anomalous diffusion in $2 \mathrm{~d}$}

In $2 \mathrm{~d}$ pores with different roughness, the diffusivity is known to decrease with increasing surface roughness $[7,28]$. As predicted from the jump length distributions discussed in section 3.1, in linear 2d pores, anomalously fast diffusion is observed [27,33], see Figs. 6(c) and (d). Since now the direction of the trajectories is determined by one rather than two angles, the relative number of jumps in directions close to the channel axis becomes much larger leading to a significantly increasing number of long jumps. As a consequence, the diffusivities $D_{s}$ and $D_{t}$ scale with an additional logarithmic factor $\ln t$, or $\ln L$, respectively. The figures have been scaled appropriately to yield a plateau for large times $t$ and large pore lengths $L$, respectively.

The logarithmic time dependence of the diffusivities vanishes when the direction of the pore axis varies along the pore length (see Fig. 8), e.g., if the elevation of the pore is shifted up or down according to a random walk or if the pore is alternating up and down. In both examples 

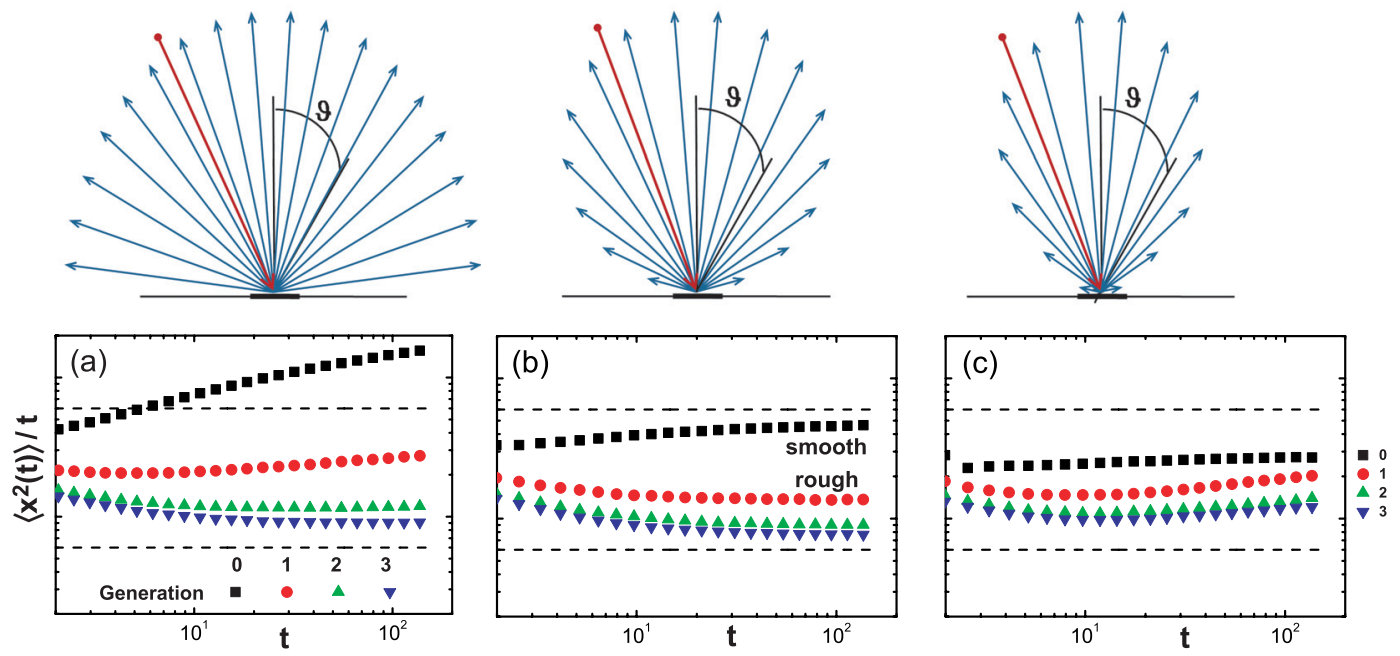

Fig. 7. (Colour online) the influence of different reflection laws (top) on the diffusion behaviour in smooth and rough $3 \mathrm{~d}$ pores. A homogeneous angular distribution results in anomalously fast diffusion in smooth pores (a); this effect is reduced by roughness (increasing from top to bottom). Diffuse reflection (Lambert's law, b) yields normal diffusion in all pores. Pronounced perpendicularly reflected particles diffuse anomalously fast in rough pores (c).

(a)

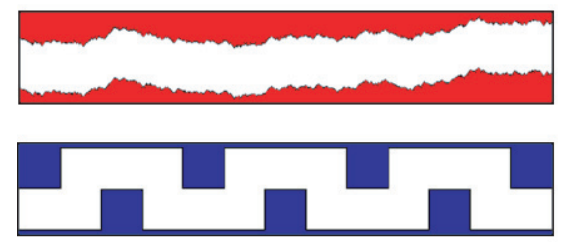

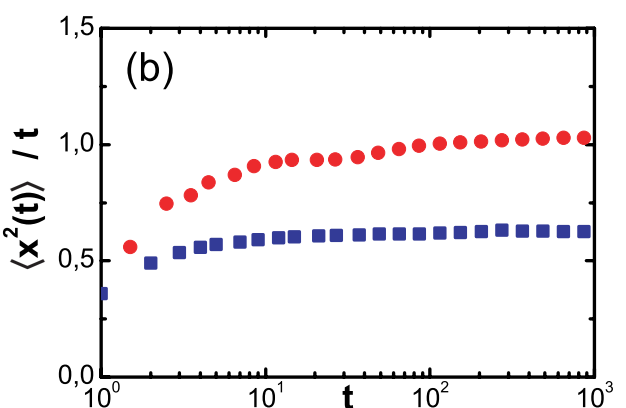

Fig. 8. (Colour online) diffusion in $2 d$ channel pores excluding the occurrence of very long jumps (a). Both for pores consisting of small channel elements with stochastically shifted axes (which, in turn, represents a random walk, circles) and for alternating pores (squares) the scaled mean-square displacement $\left\langle x^{2}(t)\right\rangle / t$ is asymptotically constant, resulting in normal diffusion behaviour (b).

the very long jumps that determine the asymptotical diffusion behaviour are suppressed, since no extremely large corridors of free flight are available. In both cases, this leads to normal diffusion.

\subsection{Analytical model}

The model assumptions (see Fig. 3) so far considered for exploring the influence of surface roughness on Knudsen diffusion exclude a straightforward analytical treatment. Such an option is provided if, deviating from the algorithm presented in Fig. 3, surface roughness is assumed to be only generated by indentations ("side pockets") in the pore walls. Figure 9(a) shows the first-order iteration step of roughness generation under such conditions. For an analytical treatment of Knudsen diffusion, the total pore space is assumed to be composed of sub-elements with the size of the indentations.

We start with estimating the reduction in diffusivity resulting from the indentations. For this purpose, we consider a fraction of the pore space, the length of which is equal to the extension of three indentations. Both pore spaces shown in Fig. 9(a) comprise two of these fractions. 
(a)

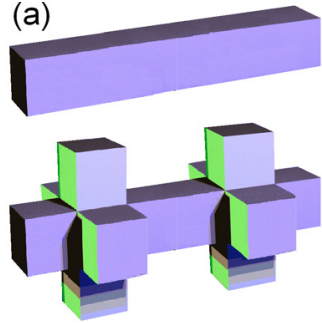

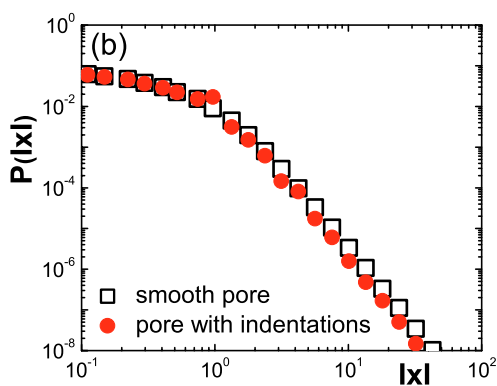

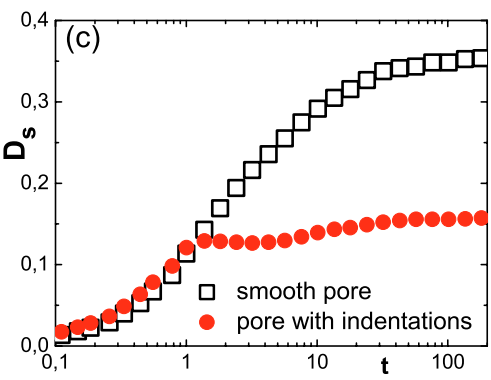

Fig. 9. (Colour online) simulations with pores accessible to analytical treatment. (a) Smooth pore and pore with indentations of $1^{\text {st }}$ order. (b) Distribution of jump lengths, differences are only observed for large jumps. (c) Smooth pore and $1^{\text {st }}$ order indented pore show the predicted difference in the self-diffusivity.

We check in which sub-element particles can move into a neighbouring sub-element. For the smooth pore a flow in $x$-direction is possible for all sub-elements. The rough pore inhibits this movement for all four added sub-elements. Only from the three original ones particles can enter the neighbours. Comparing the original pore and its first iteration with indentations should approximately yield a self-diffusivity reduced by a factor of $3 / 7: D_{s}^{1 . i t e r}=\frac{3}{7} D_{s}^{\text {smooth }}$.

Following the reasoning of section 1, in particular in the caption to Fig. 1, from the coincidence of self and transport diffusivities in the Knudsen regime $\left(D_{s}=D_{t}\right)$, the considered case of surface roughness also results in a reduced transport diffusivity by the same factor of $7 / 3$.

It is noteworthy that, in contrast to the algorithm displayed in Fig. 3, the inclusion of the present iteration procedure leads to an enhancement of the total pore space [38]. A comparison of the diffusivities as defined on the basis of Fick's First Law (Eq. (1)) has to take account of this situation. By definition [39,40], the concentrations and fluxes appearing in Eq. (1) have to refer to volume elements and areas notably exceeding the correlation lengths of the pore structure. Thus, as a result of the increasing total pore volume, the indentations shown in the bottom of Fig. 9(a) lead to an enhancement of the total number of molecules per pore element of thickness $d x$ and hence to higher concentrations (i.e., of particles per space element of thickness $d x)$. This enhancement of concentration and hence of the concentration gradient (namely by a factor of $7 / 3$ ) coincides with the decrease in the self-diffusivities. Thus, one of the pitfalls in treating such systems includes the negligence of this factor. As a result, application of Eq. (1) would lead to diffusivities which are enhanced by this very factor, which exactly compensates the diffusivity reduction by surface roughness.

Figures 9(b) and (c) display the results of our simulations for this type of pore space (Fig. 9(a)) performed with the diffusion algorithm explained in section 2. As before, we first checked the jump length distribution inside these pores. Figure 9(b) shows the expected proportionality $P(|x|) \propto|x|^{-4}$ in the long-range limit. In this range, which is decisive for the overall diffusion behaviour, the probability for the occurrence of a certain jump length in the rough pore is found to be by a factor of about $7 / 3$ smaller than in the smooth pores. Inspection of the value of $P(|x|)$ at $|x|=1$ reveals that the under-representation of long jumps in the rough pores parallels the over-representation of jumps over exactly the length of the indentations, with the latter effect resulting from the particles within these "side pockets". In the numerical simulations (Fig. 9(c)), it is this factor of $7 / 3$, by which the self-diffusivity in the rough pores is reduced in comparison with the smooth pores, in complete agreement with our above estimation. A more general derivation of an analytical formula for fractal indentations may be found in [41]. It incorporates all possible flight lengths that may occur in an indentation and correlates the expected decrease in the diffusivities with the delay inside the indentations [42].

\section{Conclusions}

We have considered molecular diffusion in channel pores with different degrees of roughness under the so-called Knudsen conditions, i.e., for negligible mutual molecular collisions and for 
flight times notably exceeding the periods of interaction with the pore walls. In particular, we have used this system to understand the differences and similarities of self diffusion and transport diffusion as the two most important situations for the measurement of molecular motion in porous materials.

We laid out the algorithm that we used in our studies in detail. The framework of the pore system is based on small cubicles wherein the successive steps of free flight or reflection are calculated. These elementary building blocks are embedded into a periodic structure to enlarge the system size. The diffusing particles are described by their velocity and three different coordinate sets, incubicle coordinate $\boldsymbol{r}$, cubicle vector $\boldsymbol{c}$ and house number $n$. The algorithm depends only on the system size and is independent of the number of walls, making it perfectly adequate for rough pore surfaces. A discrete time scale is extracted in run-time during the simulation.

At first sight, molecular migration in the $2 \mathrm{~d}$ model pores is found to deviate notably from normal diffusion, i.e. from the behaviour expected on the basis of Fick's and Einstein's diffusion equations, corroborated by the central limit theorem. This anomaly, however, may be easily rationalised by understanding the given situation of molecular dynamics as a Lévy walk. As a sufficient and necessary prerequisite for their adequacy with the relations of $1 \mathrm{~d}$ normal diffusion, the probability (density) $P(|x|)$ for the distance $x$ between two subsequent wall collisions has to scale as $P(|x|) \propto|x|^{-(1+\beta)}$ with $\beta>2$. This condition is not fulfilled for the $2 \mathrm{~d}$ model pores so that the simple case of normal diffusion does not apply. By contrast, one observes an increase of the apparent self-diffusivity with the logarithm of time, and of the apparent transport diffusivity with the logarithm of the pore length, which are nicely reflected by the numerical simulations. In the case of $3 \mathrm{~d}$ pores, reflecting a situation possibly occurring in the real nanoworld, however, normal diffusion is predicted. Only by implementing different reflection laws, that either pronounce vertically or horizontally reflected trajectories, an anomalous diffusion behaviour can also be established in the $3 \mathrm{~d}$ case. In this case, even a geometrically caused bias can be induced.

For non-interacting particles, as implied in the considered case of Knudsen diffusion, we find complete equivalence between transport diffusion and self diffusion. This is based on Fick's and Einstein's diffusion equations. The numerical simulations are in perfect agreement with these considerations. Transport diffusion and self diffusion are found to be equal with respect to both their absolute values and their dependence on the surface roughness.

Most recent progress in manufacturing silicon wafers with tubular pore structure and a large variability of the internal pore surface $[12,19,22,23]$ provide excellent conditions for an experimental verification of the simulated dependences.

The authors thankfully acknowledge funding by and stimulating discussions within the DFG-NWO sponsored International Research Training Group "Diffusion in Porous Materials", in particular with M. Kainourgiakis, F. Kapteijn, T. Steriotis, S. Vasenkov, M.-O. Coppens and A.J. Dammers are grateful to the Deutsche Forschungsgemeinschaft for financial support, as part of the "Schwerpunktprogramm SPP1155, Molekulare Modellierung und Simulation in der Verfahrenstechnik".

\section{References}

1. M. Sahimi, G.R. Gavalas, T.T. Tsotsis, Chem. Eng. Sci. 45, 1443 (1990)

2. F.J. Keil, R. Krishna, M.-O. Coppens, Rev. Chem. Eng. 16, 71 (2000)

3. J. Kärger, D.M. Ruthven, Diffusion in Zeolites and Other Microporous Solids (Wiley \& Sons, New York, 1992)

4. N.Y. Chen, T.F. Degnan, C.M. Smith, Molecular Transport and Reaction in Zeolites (VCH, New York, 1994)

5. S.B. Santra, B. Sapoval, Phys. Rev. E 57, 6888 (1998)

6. J.S. Andrade Jr., H.F. da Silva, M. Baqui, B. Sapoval, Phys. Rev. E 68, 041608 (2003)

7. K. Malek, M.-O. Coppens, Phys. Rev. Lett. 87, 125505 (2001)

8. K. Malek, M.-O. Coppens, J. Chem. Phys. 119, 2801 (2003) 
9. Handbook of Heterogeneous Catalysis, edited by G. Ertl, H. Knözinger, F. Schüth, J. Weitkamp (Wiley-VCH, Weinheim, 2008)

10. F. Schüth, K.S.W. Sing, J. Weitkamp, Handbook of Porous Solids (Wiley-VCH, Weinheim, 2002)

11. R. Krishna, B. Smit, S. Calero, Chem. Soc. Rev. 31, 185 (2002)

12. J. Kärger, R. Valiullin, S. Vasenkov, New J. Phys. 7, 1 (2005)

13. J.H. Sun, Z. Shan, Th. Maschmeyer, M.-O. Coppens, Langmuir 19, 8395 (2003)

14. S. Anandan, M. Okazaki, Microporous Mesoporous Mater. 87, 77 (2005)

15. J. Caro, M. Noack, P. Kölsch, Adsorption 11, 215 (2005)

16. E. Kierlik, P.A. Monson, M.L. Rosinberg, L. Sarkisov, G. Tarjus, Phys. Rev. Lett. 87, 055701 (2001)

17. R. Valiullin, S. Naumov, P. Galvosas, J. Kärger, H.-J. Woo, F. Porcheron, P.A. Monson, Nature 430, 965 (2006)

18. M. Dvoyashkin, R. Valiullin, J. Kärger, W.-D. Einicke, R. Gläser, J. Amer. Chem. Soc. 129, 10344 (2007)

19. A. Khokhlov, R. Valiullin, J. Kärger, F. Steinbach, A. Feldhoff, New J. Phys. 9, 272 (2007)

20. W. Feller, An Introduction to Probability Theory and its Applications (John Wiley, New York, 1970)

21. J. Kärger, Leipzig, Einstein, Diffusion (Leipziger Universitätsverlag, Leipzig, 2007)

22. B. Coasne, A. Grosman, C. Ortega, A. Simon, Phys. Rev. Lett. 88, 256102 (2002)

23. D. Wallacher, N. Künzner, D. Kovalev, N. Knorr, K. Knorr, Phys. Rev. Lett. 92, 195704 (2004)

24. S. Russ, S. Zschiegner, A. Bunde, J. Kärger, Adv. Solid State Phys. 45, 59 (2005)

25. A. Bunde, J. Kärger, S. Russ, S. Zschiegner, Diff. Fund. 2, 6 (2005)

26. S. Zschiegner, S. Russ, A. Bunde, J. Kärger, Diff. Fund. 2, 42 (2005)

27. S. Russ, S. Zschiegner, A. Bunde, J. Kärger, Phys. Rev. E 72, 030101(R) (2005)

28. S. Zschiegner, S. Russ, A. Bunde, J. Kärger, Europhys. Lett. 78, 20001 (2007)

29. Since we want to emphasize on the implementation of the algorithm, all units are set equal to 1

30. J.W. Evans, M.H. Abbasi, A. Sarin, J. Chem. Phys. 72, 2967 (1980)

31. S. Zschiegner, S. Russ, A. Bunde, J. Kärger, Diff. Fund. 4, 13 (2007)

32. J. Klafter, M.F. Shlesinger, G. Zumofen, Phys. Today 49, 33 (1996)

33. P. Levitz, Europhys. Lett. 39, 593 (1997)

34. D. Ben-Avraham, S. Havlin, Diffusion and Reactions in Fractals and Disordered Systems (Cambridge University Press, Cambridge, 2000)

35. To be comparable to other algorithms, all results are shown in rescaled units: the unit of length is one pore diameter, the particle velocity is fixed to one pore diameter per time unit and thus specifies the unit of time

36. G. Zumofen, J. Klafter, Phys. Rev. E 47, 851 (1993)

37. A.J. Dammers, M.-O. Coppens, Proceedings of the $7^{\text {th }}$ World Congress of Chemical Engineering (Glasgow, 2005)

38. K. Malek, M.-O. Coppens, Coll. Surf. A 206, 335 (2002)

39. J. Kärger, S. Vasenkov, Microporous Mesoporous Mater. 85, 195 (2005)

40. J. Kärger, in [9], p. 1714

41. M.-O. Coppens, G.F. Froment, Fractals 3, 807 (1995)

42. M.-O. Coppens, A.J. Dammers, Fluid Phase Equil. 241, 308 (2006) 